\title{
Functional Asymmetries in ON and OFF Ganglion Cells of Primate Retina
}

\author{
E. J. Chichilnisky and Rachel S. Kalmar \\ Systems Neurobiology, The Salk Institute, La Jolla, California 92037, and University of California, San Diego, La Jolla, \\ California 92037
}

Functional asymmetries in the ON and OFF pathways of the primate visual system were examined using simultaneous multi-electrode recordings from dozens of retinal ganglion cells (RGCs) in vitro. Light responses of RGCs were characterized using white noise stimulation. Two distinct functional types of cells frequently encountered, one ON and one OFF, had nonopponent spectral sensitivity, relatively high response gain, transient light responses, and large receptive fields (RFs) that tiled the region of retina recorded, suggesting that they belonged to the same morphological cell class, most likely parasol. Three principal functional asymmetries were observed. (1) Receptive fields of ON cells were $20 \%$ larger in diameter than those of OFF cells, resulting in higher full-field sensitivity. (2) ON cells had faster response kinetics than OFF cells, with a 10$20 \%$ shorter time to peak, trough and zero crossing in the biphasic temporal impulse response. (3) ON cells had more nearly linear light responses and were capable of signaling decrements, whereas OFF cells had more strongly rectifying responses that provided little information about increments. These findings suggest specific mechanistic asymmetries in retinal $\mathrm{ON}$ and OFF circuits and differences in visual performance on the basis of the activity of ON and OFF parasol cells.

Key words: retinal ganglion cell; receptive field; monkey; retina; kinetics; dynamics; sensitivity; white noise; nonlinear
The ON and OFF pathways of the visual system (Hartline, 1938; Kuffler, 1953) are prototypical examples of parallel processing in neural circuits. ON-OFF segregation begins with the divergence of photoreceptor signals to sign-conserving and sign-inverting second order (bipolar) retinal neurons (Werblin and Dowling, 1969) and is preserved in the brain. These pathways have generally been treated as symmetric systems with equal and opposite light responses that primarily transmit information about increments and decrements of light, respectively (for review, see Schiller, 1992). For example, the parasol retinal ganglion cells in primates that project to the magnocellular layers of the lateral geniculate nucleus are composed of morphologically and physiologically similar ON and OFF types, with opposite sign light responses and dendritic fields (DFs) that tile the retina (Polyak, 1941; Watanabe and Rodieck, 1989; Silveira and Perry, 1991; Dacey and Brace, 1992). However, some studies have suggested that the ON and OFF pathways are not fully symmetric. Psychophysical evidence has indicated asymmetries in perception and detection of incremental and decremental stimuli (Bowen et al., 1989; Wehrhahn and Rapf, 1992; Kremers et al., 1993), although it is unclear whether psychophysical methods can truly isolate the $\mathrm{ON}$ and OFF pathways or at what point in the visual system the observed asymmetries arise. Anatomical evidence indicates that the dendritic fields of parasol (human) and $\alpha$ (rat, dog) ON retinal

Received July 16, 2001; revised Jan. 7, 2002; accepted Jan. 8, 2002.

This work was supported by National Institutes of Health Grant EY-13150, a Sloan Research Fellowship, a McKnight Scholar's Award (E.J.C.), and a University of California, San Diego Undergraduate Research Scholarship (R.S.K.). We thank E. Callaway, S. Zola, and T. Albright for providing access to tissue, D. Baylor, E. Callaway, J. Demb, S. du Lac, and F. Rieke for useful discussions, A. Litke and colleagues for technology development, D. Chander for assistance during experiments, and S. Barry and R. Roder for technical assistance.

Correspondence should be addressed to E. J. Chichilnisky, Systems Neurobiology, The Salk Institute, 10010 North Torrey Pines Road, La Jolla, CA 92037-1099. E-mail: ej@salk.edu.

Copyright (C) 2002 Society for Neuroscience $0270-6474 / 02 / 222737-11 \$ 15.00 / 0$ ganglion cells are larger than their OFF counterparts (Peichl et al., 1987; Peichl, 1989; Dacey and Petersen, 1992; Tauchi et al., 1992), suggesting asymmetries in receptive field (RF) size. However, electrophysiological studies have revealed little evidence of functional asymmetries in the ON and OFF pathways (Linsenmeier et al., 1982; Kremers et al., 1993; Benardete and Kaplan, 1997, 1999; Lankheet et al., 1998).

Using multi-electrode recordings, we demonstrate significant asymmetries in spatial summation, kinetics, nonlinearity, and sensitivity in light responses of simultaneously recorded ON and OFF ganglion cells of the macaque monkey retina. The ensembles of ON and OFF cells recorded tiled the same retinal area and apparently represented the same morphological class, most likely parasol. ON-OFF asymmetries were consistent within and across experimental preparations. Receptive field size asymmetries were consistent with known asymmetries in dendritic fields. Kinetic asymmetries could reflect distinct mechanisms governing the undershoot of biphasic light responses. Nonlinearity asymmetries suggest differences in spike threshold or basal transmitter release in $\mathrm{ON}$ and $\mathrm{OFF}$ retinal neurons. Thus the $\mathrm{ON}$ and $\mathrm{OFF}$ pathways display significant functional asymmetries that originate in the retinal circuitry and may influence visual sensitivity and perception.

\section{MATERIALS AND METHODS}

Preparation. Eyes were obtained from terminally anesthetized macaque monkeys (Macaca fascicularis, M. mulatta, M. radiata) used by other experimenters, in accordance with institutional guidelines for the care and use of animals. Immediately after enucleation, the anterior portion of the eye and vitreous were removed in room light, and the eye cup was placed in bicarbonate-buffered Ames' solution (Sigma, St. Louis, MO) and stored in darkness for at least $20 \mathrm{~min}$ before dissection. Under infrared illumination, pieces of retina $2-4 \mathrm{~mm}$ in diameter were cut from regions $10-40^{\circ}$ from the fovea and placed flat against a planar array of 61 extracellular microelectrodes that were used to record action potentials from retinal ganglion cells (Meister et al., 1994; Chichilnisky and Baylor, 
1999a). The preparation was superfused with Ames' solution bubbled with $95 \% \mathrm{O}_{2}$ and $5 \% \mathrm{CO}_{2}$ and maintained at $35-36^{\circ} \mathrm{C}, \mathrm{pH}$ 7.4. In most experiments the piece of retina was separated from the retinal pigment epithelium (RPE) before recording. In 5 of 13 preparations the RPE was left attached. Results from RPE-attached preparations were similar to results from isolated retina preparations.

Retinal eccentricity of some preparations was measured with a precision of 1-2 $\mathrm{mm}$. Eccentricities are expressed below as temporal equivalent, because the contours of constant RGC density (and thus presumably dendritic and receptive field size) in the macaque monkey retina are approximately semicircular in the temporal half of the retina, but elliptical with an aspect ratio of 0.61 in the nasal half (Perry and Cowey, 1985; Watanabe and Rodieck, 1989). Thus a location $X \mathrm{~mm}$ nasal and $Y \mathrm{~mm}$ superior (or inferior) to the fovea was assigned an equivalent eccentricity of $\sqrt{(X / 0.61)^{2}+Y^{2}}$. A location $X \mathrm{~mm}$ temporal and $Y \mathrm{~mm}$ superior (or inferior) to the fovea was assigned an equivalent eccentricity of $\sqrt{X^{2}+Y^{2}}$. Visual angle $(A)$ in degrees from previous studies (Croner and Kaplan, 1995) was converted to retinal eccentricity in millimeters $(E)$ by inverting the relation $A=0.1+4.21 E+0.038 E^{2}$ (Drasdo and Fowler, 1974; Dacey and Petersen, 1992).

Stimuli. The preparation was stimulated with the optically reduced (1.0-1.3 mm diameter) image of a cathode ray tube computer display refreshing at 66.67 or $120 \mathrm{~Hz}$, focused on the photoreceptor layer by a microscope objective, and centered on the 480- $\mu$ m-diameter electrode array. Stimuli were attenuated to low photopic light levels using neutral density filters. In isolated retina experiments the stimulus was delivered from the photoreceptor side. In experiments in which the RPE was attached, the preparation was stimulated from the retinal ganglion cell side through the mostly transparent electrode array. In the latter case the shadows cast by the platinized (black) electrode tips, $5 \mu \mathrm{m}$ in diameter and spaced $60 \mu \mathrm{m}$ apart, had a minimal influence on the intensity and spatial pattern of the stimulus, because they occupied roughly $1 \%$ of the total area of the array and were optically diffused by virtue of lying in a different focal plane than the photoreceptors.

The stimulus consisted of a square lattice of randomly flickering pixels that was presented for $15-45$ min. Random flicker was created by selecting the intensities of the red, green, and blue display phosphors at each pixel location independently from a Gaussian or binary (twovalued) distribution every $15 \mathrm{msec}(66.67 \mathrm{~Hz}$ display) or $8.33 \mathrm{msec}(120$ $\mathrm{Hz}$ display). This stimulus modulated photon absorptions asynchronously in all three cone types. Pixel size varied between 24 and $72 \mu \mathrm{m}$ at the retina in different experiments. The rms contrast of the three phosphors on the display varied between 0.32 and 0.96 ; in each experiment the rms contrast of all three phosphors was equal.

The typical mean photon absorption rate for the long, middle, and short wavelength sensitive cones was approximately equal to the absorption that would have been caused by spatially uniform monochromatic lights of wavelength 561, 530, and $430 \mathrm{~nm}$ and intensity 9200,9200 , and 5100 photons per $\mu \mathrm{m}^{-2} / \mathrm{sec}^{-1}$, respectively, incident on the photoreceptors. For RPE-attached preparations, this intensity includes a factor of 2 for the light-funneling effect of the inner segments (Packer et al., 1996). In some preparations the intensity was roughly double or half the above.

Recordings. Spikes were digitized at $20 \mathrm{kHz}$ (Meister et al., 1994; Litke, 1999) and stored for off-line analysis. Spikes from 15-85 cells were segregated by identifying distinct clusters of spike height and width recorded on each electrode and verifying the presence of a refractory period. For quantitative analysis of light responses, spike counts from each cell were computed in time bins of $15 \mathrm{msec}(66.67 \mathrm{~Hz}$ display) or $8.33 \mathrm{msec}(120 \mathrm{~Hz}$ display).

Model of light responses. Analysis of visual signaling required a quantitative model of RGC light response that, unlike classical models, accounts for significant nonlinearities demonstrated below. Light responses were characterized using a linear-nonlinear (LN) cascade model for firing rate as a function of the stimulus [for a description of the model and analysis, see Korenberg and Hunter (1986), Chichilnisky (2001); for a test of the validity of the model in the present experimental conditions, see Chichilnisky (2001), Kim and Rieke, (2001)]. Briefly, it is assumed that (1) contrast modulations over space and time are pooled linearly to create a generator signal, and (2) spike rate at each point in time is determined by a (generally nonlinear) function of the generator signal at the same point in time. The parameters of this model are as follows: $w$, the linear weighting of the stimulus over space and recent time that creates the generator signal, and $N$, the function that transforms the generator signal to spike rate. Thus, if $s$ is a vector the entries of which represent the contrast of each phosphor at each spatial location over recent time, the instantaneous firing rate is given by:

$$
R(s)=N(w \cdot s),
$$

where $\cdot$ is the inner product of vectors. Under the assumptions of the LN model, it can be shown that $w$ is equal to the spike-triggered average (STA) stimulus, that is, the average stimulus over a period of time preceding a spike (Chichilnisky, 2001). This period was chosen empirically to exceed the duration of the impulse response of the cell. To complete the model for light response required only obtaining an estimate for $N$. The generator signal at each time during stimulation was estimated by summing the elements of the recent stimulus multiplied by a parametric fit to the STA (to reduce estimation bias; see below). The spike rate associated with each distinct value of the generator signal was obtained by averaging spike counts over many time points in which nearly the same value of the generator signal was observed. This procedure, repeated over the range of observed values of the generator signal, yielded the relationship between generator signal and average spike rate, that is, the function $N$. This completes the model for light response. Examples of the STA and nonlinearity for one cell are shown in Figure $1 A$.

Fitting and parameter estimation. Together, the weighting of stimuli over space and time ( $w$, equal to the STA) and the response nonlinearity $(N)$ provide a description of the average response to any stimulus. Spatial, kinetic, and sensitivity measures were obtained from smooth functional approximations to $w$ and $N$. The former was accurately described as the product of a spatial sensitivity function, a temporal sensitivity function, and a chromatic sensitivity function. The spatial sensitivity function was defined as a difference of two-dimensional Gaussian profiles (Rodieck, 1965) with common elliptical isosensitivity contours, representing the center and surround of the RF:

$$
s(v)=e^{-\frac{1}{2}(v-u)^{T} Q(v-u)}-k e^{-\frac{1}{2} r(v-u)^{T} Q r(v-u)} .
$$

Here $v$ is two-dimensional vector that specifies a spatial location, $s(v)$ indicates the sensitivity at that spatial location, $u$ is a two-dimensional vector that specifies the midpoint of the RF, $Q$ is a $2 \times 2$ symmetric positive semi-definite matrix that specifies the elliptical Gaussian shape of the RF center, $k$ is a scalar that specifies the relative strength of the surround, and $1 / r$ is a scalar that specifies the relative size of the surround.

The temporal sensitivity function specifies how strongly the stimulus contrast at a time $t$ before the present influences firing rate. This was given by the difference of two cascades of low-pass filters:

$$
f(t)=p_{1}\left(t / \tau_{1}\right)^{n} e^{-n\left(t / \tau_{1}-1\right)}-p_{2}\left(t / \tau_{2}\right)^{n} e^{-n\left(t / \tau_{2}-1\right)} .
$$

Here, $t$ specifies time before the present, $f(t)$ is the sensitivity at that time, and $n, p_{1}, p_{2}, \tau_{1}$, and $\tau_{2}$ are free parameters.

Finally, chromatic sensitivity was captured by two additional scalars representing the relative sensitivity to modulation of the three phosphors (the time courses of the three phosphors in the STA were always very nearly in a scalar relationship, consistent with dominant $\mathrm{L}$ and $\mathrm{M}$ cone input; see Figs. 2, 8). The product of the spatial, temporal, and chromatic sensitivities defined above determined the fit to the STA (i.e., $w$ ).

The response nonlinearity $N$ was well approximated using the lower portion of a sigmoidal function:

$$
n(x)=a G(b x-c),
$$

where $x$ is the generator signal, $n(x)$ is the firing rate, $G(x)$ is the cumulative normal (indefinite integral of standard normal distribution), and $a, b$, and $c$ are free parameters.

Together, these fits to $w$ and $N$ provide a full parametric model of light response (see Eq. 1). In a typical measurement such as that in Figure $1 A$, the STA was obtained over a $30 \times 30$ spatial grid with three colors and 30 time bins $(250 \mathrm{msec})$ per location, for a total of 81,000 values. The model fit, shown in Figure $1 B$, described this STA with 14 parameters The nonlinearity was described by three parameters. Parameters were selected to minimize mean squared error using Powell's method (Press et al., 1988). Estimates of peak, trough, and zero crossing of the STA time course, RF location, and value and slope of nonlinearity were taken from these fits. RF diameter was defined as the diameter of a circle with the same area as the $1 \mathrm{SD}$ (elliptical) boundary of the Gaussian center profile. RF integration area was defined as $2 \pi$ times the square of the diameter, that is, the volume under the Gaussian center profile. Usually, spatially antagonistic surrounds were weak relative to centers (Fig. 1); 
A

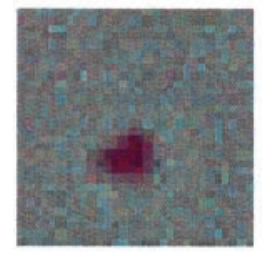

B

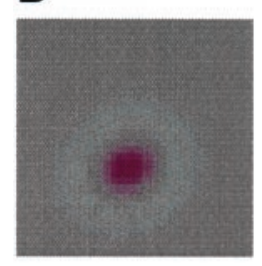

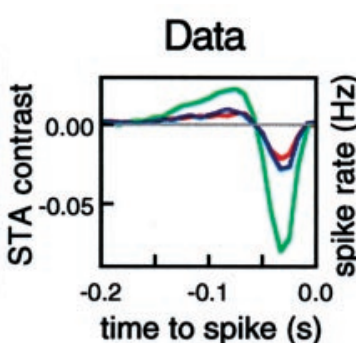

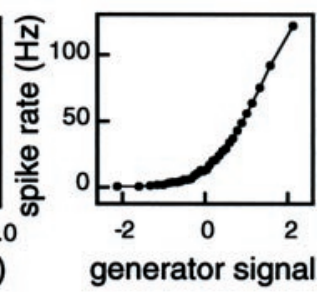

Model

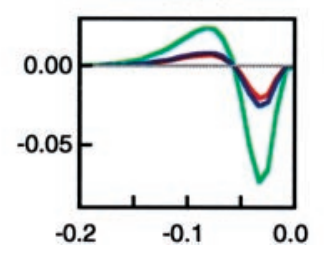

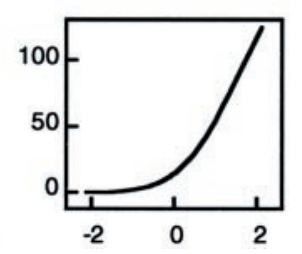

Figure 1. Characterization of light response and parametric fits. A, Left panel, The average stimulus observed $33 \mathrm{msec}$ (4 frames, near time-topeak) before a spike in one RGC. The dark central region reveals the receptive field of the cell. Middle panel, Average time course of contrast of the red, green, and blue display phosphors in the $250 \mathrm{msec}$ preceding a spike, summed over 36 pixels in the center of the receptive field. The dominant negative lobe indicates that this is an OFF cell. Right panel, Average firing rate as a function of the generator signal (stimulus weighted by STA) observed during white noise stimulation. $B$, Parametric fits, as described in Materials and Methods, to the corresponding panels in $A$.

results obtained with single Gaussian spatial profiles $(k=0)$ yielded the same conclusions in all analyses presented below.

Statistical comparison of each light response parameter (e.g., RF diameter) for ON and OFF cells was performed by computing the mean value of the parameter for ON and OFF cells in each preparation, $\mu_{o n}$ and $\mu_{\text {off }}$, dividing each mean by the pooled sample SD $\sigma$ to obtain a normalized value, and then performing a two-tailed nonparametric Wilcoxon order test (Rice, 1988) on pairs of the form $\left(\mu_{o n} / \sigma, \mu_{o f f} / \sigma\right)$ accumulated from multiple preparations. Whenever possible, parameters obtained directly from raw data were examined to check that fits did not introduce systematic errors. In all cases, raw data and fits yielded the same pattern of results.

\section{RESULTS}

\section{Characterization of light response}

Retinal ganglion cells were characterized and classified on the basis of their responses to white noise stimulation (Sakai et al., 1988; Chichilnisky, 2001). The stimulus was a square lattice of randomly flickering pixels with no spatial, temporal, or chromatic structure. The light response properties of each cell were summarized by the spike-triggered average stimulus (STA). The STA is a measure of how effectively stimuli at different locations and with different colors are integrated by the cell over time to control firing (see Materials and Methods).

STAs from six simultaneously recorded cells are shown in Figure 2. For each cell, the average stimulus $33 \mathrm{msec}$ before a spike is displayed as an image. The spatial RF of each cell is indicated by the region of the image that deviates from the gray background. The cells on the left (right) of Figure 2 were predominantly excited by increases (decreases) in the intensity of the three phosphors and were therefore classified as ON (OFF) cells. Antagonistic RF surrounds are present in these images, although they are weaker than the centers. The second panel for each cell shows the time course of red, green, and blue phosphor intensities preceding a spike, summed over the pixels in the RF center.

These biphasic time courses indicate how the cell integrated visual inputs of different colors over recent time.

The STA alone would provide a full description of RGC light responses if responses were linear. Because they generally were not, a simple nonlinear model was used to obtain a more accurate, quantitative characterization (see Materials and Methods for details). In the model it is assumed that (1) contrast modulations over space and time are pooled linearly to create a generator signal, and (2) spike rate at each point in time is given by a (generally nonlinear) function of the generator signal at the same point in time. Under these conditions it can be shown that the STA reveals the linear weighting (Chichilnisky, 2001). The relation between generator signal and firing rate can be determined by comparing the STA-weighted stimulus to observed spike counts. Examples are shown in the third panel for each cell in Figure 2. If responses were linear, these data would fall on straight lines; the departure from this prediction highlights the importance of using a nonlinear model to characterize light response. The above model can be used to predict the response to any stimulus, and empirical tests indicate that it describes light responses fairly accurately in the present conditions (Chichilnisky, 2001; Kim and Rieke, 2001). Importantly, because this model allows for response nonlinearities such as rectification and saturation, it makes weaker assumptions about light responses than commonly used strictly linear models.

\section{Cell classification}

Because distinct morphological cell classes have different light responses, an analysis of ON-OFF asymmetries is only meaningful for opposite sign cells of the same morphological class, e.g., parasol. A category of cells was identified on the basis of RF size, response kinetics, response gain, and tiling that apparently represents a single morphological class, most likely parasol. The ON and OFF cells in Figure 2 are examples.

Cell classification was performed as follows. Blue-on/yellow-off cells in each preparation were easily identified on their coloropponent STA time courses (Chichilnisky and Baylor, 1999a). Cells with opposite color opponency (blue-off/yellow-on) were not observed, so these cells were excluded from further analysis. Among the remaining cells, four distinct functional groups were routinely identified by their stereotyped light response properties. Figure 3 shows such a classification in two preparations. Scatter plots show the RF diameter and the peak amplitude of the STA for each cell: ON cells fall on the right (positive STA peak) and OFF cells on the left (negative STA peak). Within the ON and OFF groups, clusters of cells with large and small RF sizes are evident; large RF clusters are identified in the figure. Because the anatomical identities of these cell groups are uncertain, they will be referred to as the large (L)-ON, small (S)-ON, L-OFF, and S-OFF cells. The RFs of the L-ON and L-OFF groups often partially tiled the area of retina recorded. In Figure 3, outlines of the RFs of all L-OFF and L-ON cells from each preparation are shown above the scatter plots, superimposed on the hexagonal boundary of the electrode array. These RFs closely abutted with little overlap; neighboring RFs were separated by 1-1.5 RF diameters. This tiling did not simply reflect the regular spacing of electrodes because tiling with neighbor distances of 100-300 $\mu \mathrm{m}$ was observed in different preparations, whereas inter-electrode spacing was always $60 \mu \mathrm{m}$.

Taken together, the clustering and tiling of L-ON and L-OFF RFs suggest that each of these cell groups corresponded to a single morphological cell type, similar to the tiling reported in 
ON
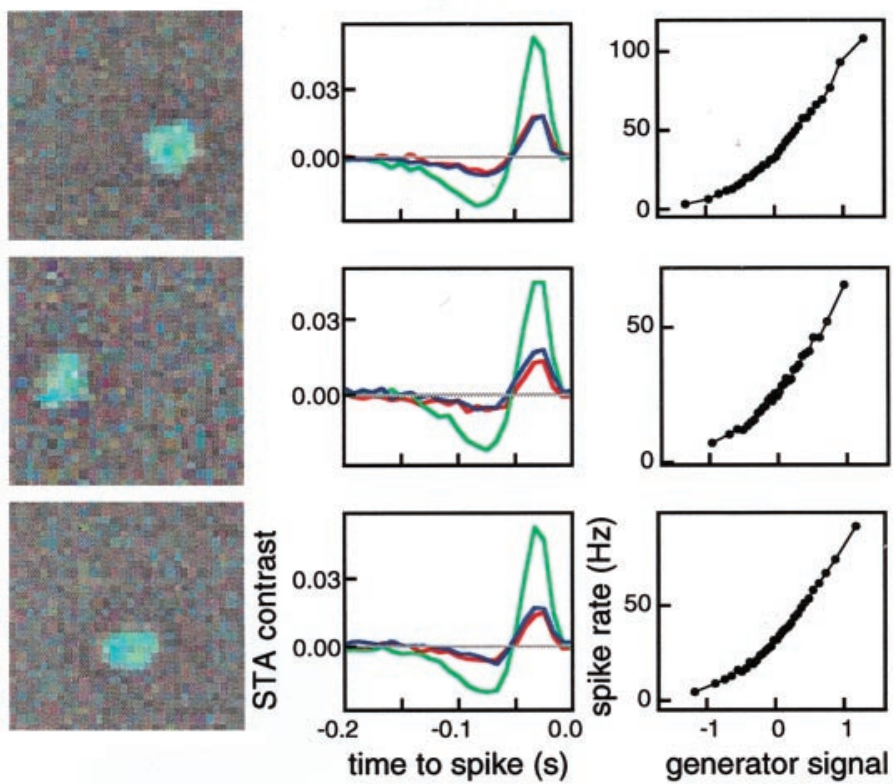
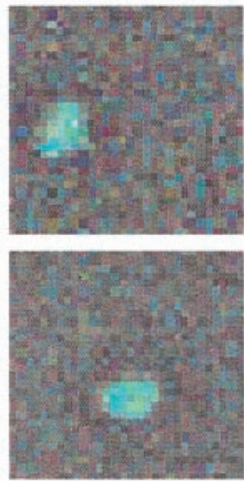

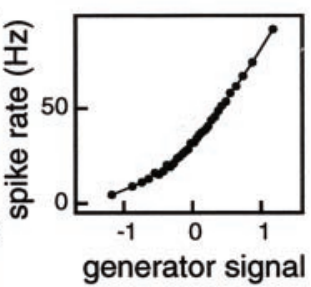

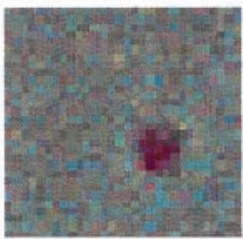
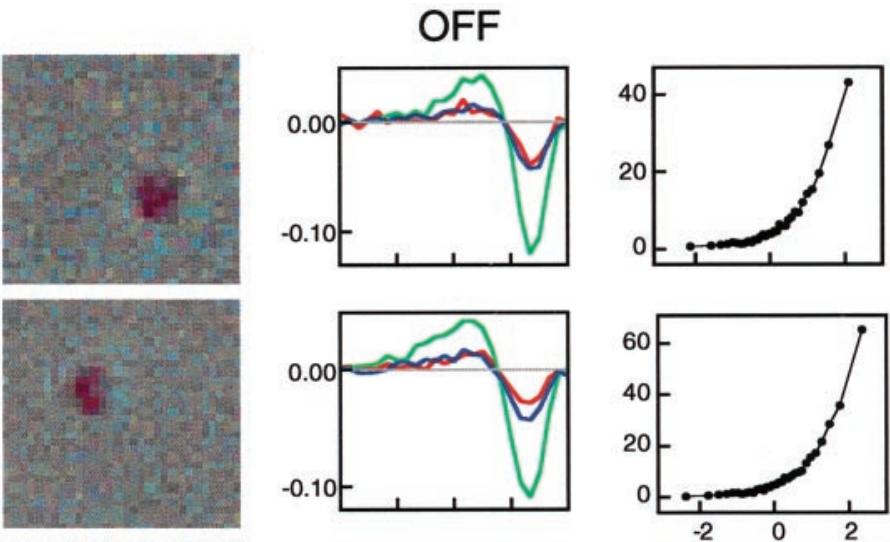

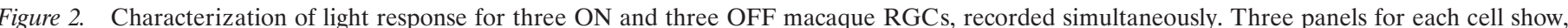

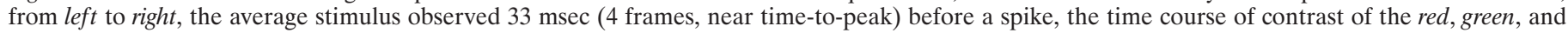

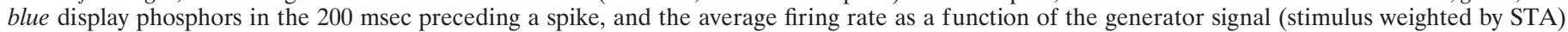
observed during white noise stimulation, in the same format as Figure $1 \mathrm{~A}$.
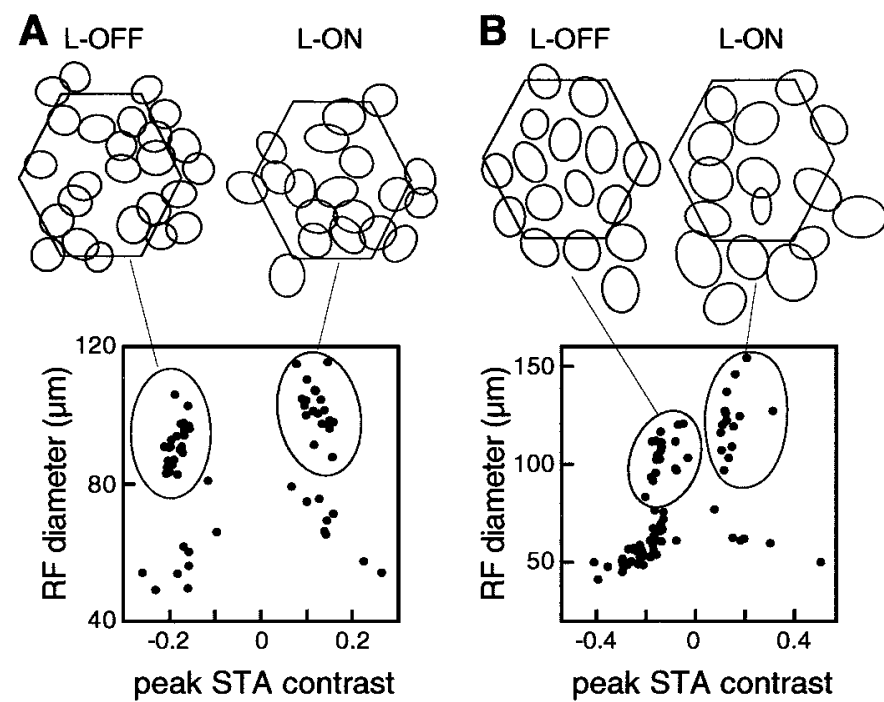

Figure 3. Cell classification for two preparations $(A, B)$. $A$, Scatter plot shows the RF diameter and peak STA contrast for each of 62 cells recorded simultaneously. Clusters defining L-ON cells (right) and L-OFF cells (left) are indicated by ovals. Top panel shows outlines of RFs (1 SD boundary of Gaussian fit; see Materials and Methods) for all L-OFF cells and L-ON cells in this preparation. $B$, Data from 85 cells recorded in a second preparation, in the same format as $A$.

rabbit retina (Devries and Baylor, 1997), which probably reflected complete non-overlapping coverage of the retina by the dendritic fields of cells of each type (Wassle et al., 1981). Two observations further suggest that the L-ON and L-OFF groups are of the same morphological class, e.g., parasol. First, the L-ON and L-OFF cells were more similar to one another in RF size, kinetics, response gain (Fig. 4), and chromatic sensitivity than to simultaneously recorded S-ON, S-OFF, and blue-on/yellow-off cells.
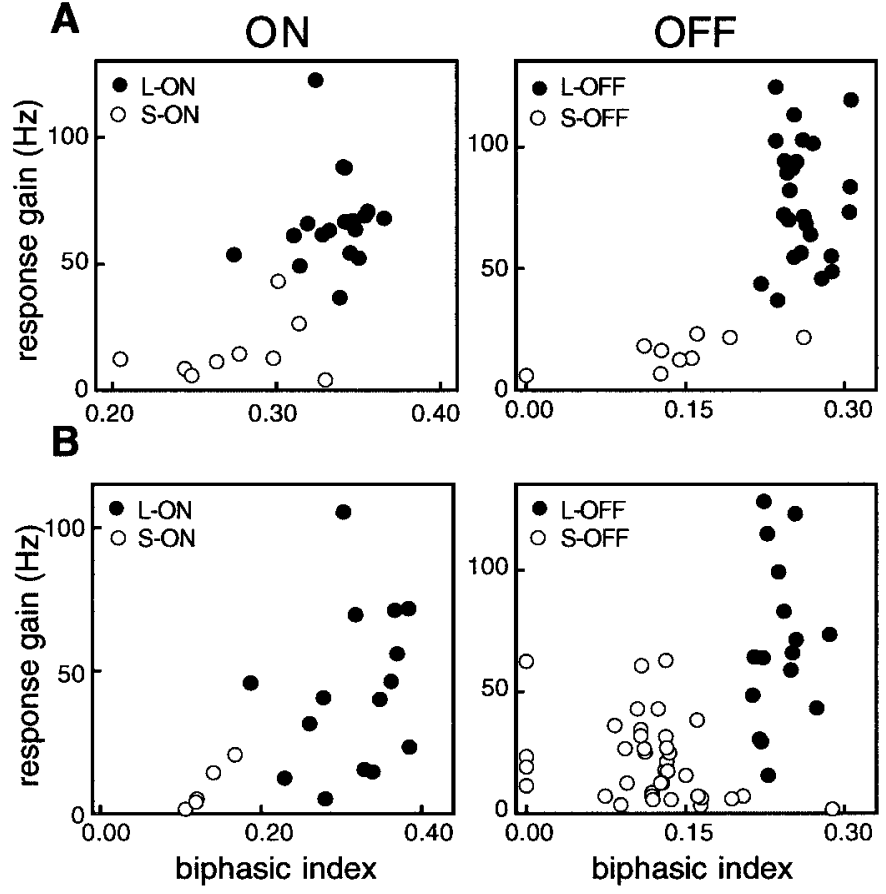

Figure 4. Kinetics and response gain for cells with large and small RFs, for two preparations $(A, B)$. A, Left, Response gain (derivative of spike rate with respect to contrast of an achromatic $15 \mathrm{msec}$ full-field flash, deduced from white noise measurements) and index of biphasicity (absolute value of ratio of trough to peak of STA time course) for all L-ON and all S-ON cells in the preparation of Figure $3 A$. L-ON cells are shown by filled symbols; S-ON cells are shown by open symbols. Right, Same measurements for all L-OFF and S-OFF cells in the same preparation. L-OFF cells are shown by filled symbols; S-OFF cells are shown by open symbols. $B$, Same as $A$, for the data set from Figure $3 B$. 


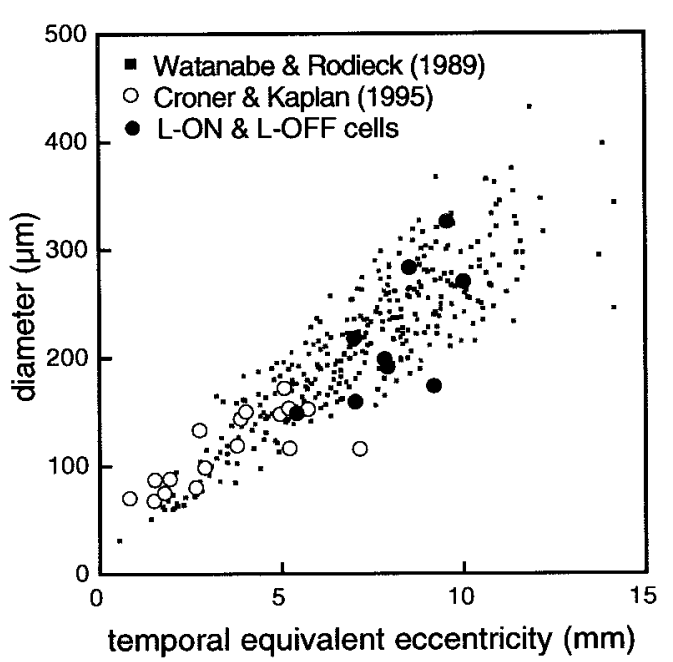

Figure 5. Comparison of L-ON and L-OFF cells to parasol cells. Small squares show DF field diameters of individual parasol cells as a function of retinal eccentricity, replotted from Watanabe and Rodieck (1989). Open circles show RF diameters of individual magnocellular-projecting RGCs, replotted from Croner and Kaplan (1995), multiplied by 1.57, a value chosen by linear regression to bring RF diameters into registry with DF diameters. Filled circles show mean RF diameters of all L-ON and L-OFF cells from each of nine preparations, also multiplied by 1.57 .

Second, consistent clustering of cell groups in different preparations (Fig. 3) suggests that the L-ON and L-OFF groups represent each cell type commonly observed, probably because their morphological and electrotonic properties led to favorable sampling by the multi-electrode arrays used. Presumably, their opposite-sign morphologically similar counterparts would also be sampled frequently, particularly in the peripheral retina where ganglion cell bodies form a monolayer and cell-type specific somatic lamination (Perry and Silveira, 1988) cannot introduce additional sampling biases. Thus it is likely that L-ON and L-OFF cells are of the same class. Two observations further suggest that the L-ON and L-OFF cells are parasol cells (Polyak, 1941).

First, L-ON and L-OFF cells had higher response gain and more biphasic, or transient, light responses than their S-ON and S-OFF counterparts. The left panel of Figure $4 A$ shows response gain as a function of an index of the biphasicity of the STA for the L-ON and S-ON cells shown in Figure $3 A$. The right panel shows the same plot for L-OFF and S-OFF cells from the same preparation. L-ON and L-OFF cells generally had higher response gain and more biphasic light responses than simultaneously recorded $\mathrm{S}-\mathrm{ON}$ and S-OFF cells, respectively. Figure $4 B$ shows the same trend for the cells of Figure $3 B$. Similar results were observed for L-ON and S-ON cells in seven of eight other preparations; few $\mathrm{S}-\mathrm{OFF}$ cells were observed in other preparations.

Second, L-ON and L-OFF cells had RF diameters that would be expected for parasol cells at the same retinal eccentricity. Because no survey exists of parasol cell RF diameters as a function of eccentricity, this was determined as follows. The small squares in Figure 5 show dendritic field diameters of parasol cells as a function of retinal eccentricity (Watanabe and Rodieck, 1989). The open circles show RF diameters (twice the SD of Gaussian fits) of magnocellular-projecting RGCs recorded in vivo (Croner and Kaplan, 1995) that are presumably mostly parasol cells (Lee, 1996). Because it is unclear how RF diameters measured this way should compare with DF diameters, the latter have been scaled to bring the RF diameters into registry with DF diameters. Finally, filled circles indicate the mean L-ON and L-OFF RF diameters from nine preparations in which eccentricity information was available, scaled by the same factor. These values fall within the distribution of parasol DFs.

The differences in response gain and kinetics in Figure 4 would be expected (Lee, 1996) if L-ON and L-OFF cells corresponded to parasol cells and S-ON and S-OFF cells corresponded to midget cells [note that midget cells in the peripheral retina do not display color opponency (Dacey, 2000)]. The receptive field diameters in Figure 5 would be expected if L-ON and L-OFF cells were parasol cells. Furthermore, simultaneously recorded blueon/yellow-off cells had RF diameters comparable to those of L-ON and L-OFF cells (Chichilnisky and Baylor, 1999a), and S-ON and S-OFF cell RFs were roughly half as large (Fig. 3). These proportions roughly match the relative DF diameters of the midget, parasol, and small bistratified cells that together constitute a majority of RGCs (Perry et al., 1984; Watanabe and Rodieck, 1989) and are frequently encountered with extracellular electrodes (Lee, 1996). In the peripheral primate retina, roughly $45 \%$ of all RGCs are midgets, $20 \%$ are parasols, and $10 \%$ are small bistratified (Dacey, 1994). Thus for the L-ON and L-OFF cells to be other than parasols would require the presence of another morphological class of RGC with RF size and density similar to parasols, constituting a majority of the remaining $25 \%$ of RGCs in the peripheral retina. A cell class of this density has not been reported.

It is assumed in what follows that the L-ON and L-OFF cells defined above are of the same morphological class, most likely parasol. The visual signaling properties of L-ON and L-OFF cells differed in three principal respects: RF size, kinetics, and linearity.

\section{Larger receptive fields in ON cells}

L-ON cells had consistently larger RFs than L-OFF cells. This can be seen in Figure 3, where for each preparation the distribution of L-ON cell RF sizes is slightly higher than that of L-OFF cell RF sizes. The asymmetry in RF size can be seen directly in Figure 6, which shows the RFs of nine L-ON and nine L-OFF cells recorded simultaneously in one preparation. All L-OFF cells recorded are shown, and the nine L-ON cells shown uniformly span the range from largest to smallest L-ON RF size recorded. Both groups of cells are sorted by RF size. Clearly, as would be predicted from the plots in Figure 3, the distribution of RF sizes of L-ON cells and L-OFF cells overlaps significantly. However, when the largest (top row), intermediate (middle row), and smallest (bottom row) RFs in Figure 6 are compared, it is clear that L-ON cells had, on average, slightly larger RFs than L-OFF cells. In this preparation, the mean $( \pm$ SEM) RF diameter for L-ON cells was $100( \pm 3.2) \mu \mathrm{m}$ and for L-OFF cells was $87( \pm 4.5) \mu \mathrm{m}$. Figure 2 shows another example of RF size asymmetry. This trend was consistent across preparations. Each point in Figure $7 A$ shows the mean and SEM of L-ON and L-OFF RF sizes in one preparation; 17 preparations are represented. The points fall below the identity diagonal, indicating larger L-ON cell RFs. A linear regression to these data indicates that L-ON cells had, on average, $21 \%$ larger RF diameters than L-OFF cells $(p<0.001$; see Materials and Methods).

Because RF size was estimated from Gaussian fits, deviations of actual RF profiles from an idealized Gaussian shape could bias RF size estimates and artifactually indicate asymmetries. Two considerations argue against this. First, spatially antagonistic sur- 
ON
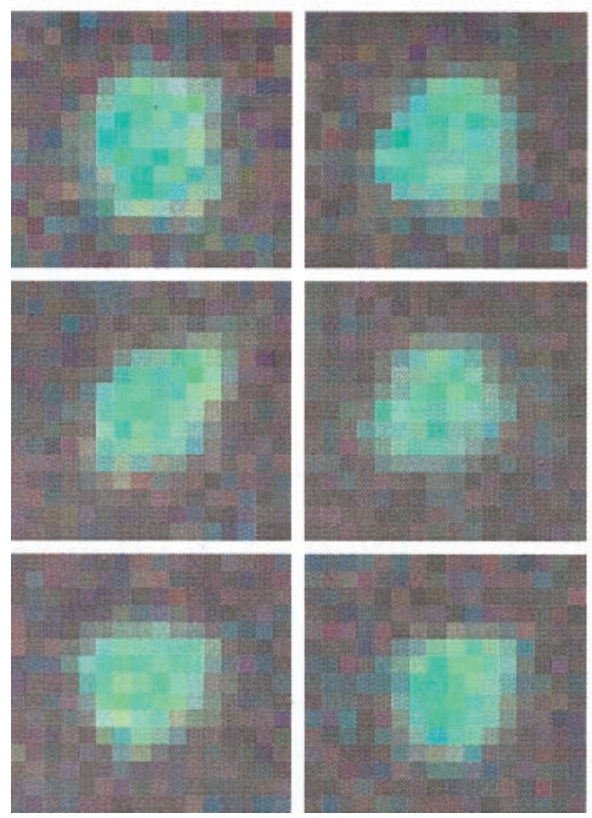
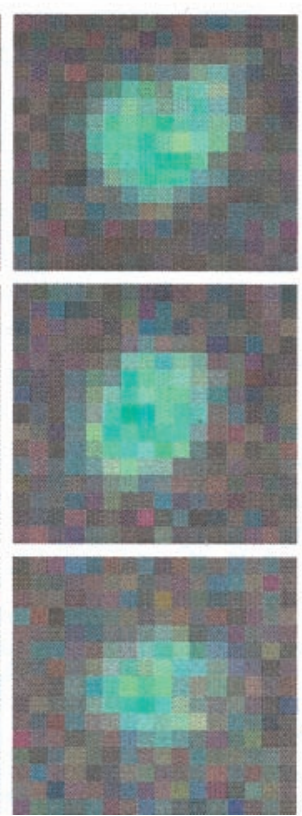

OFF
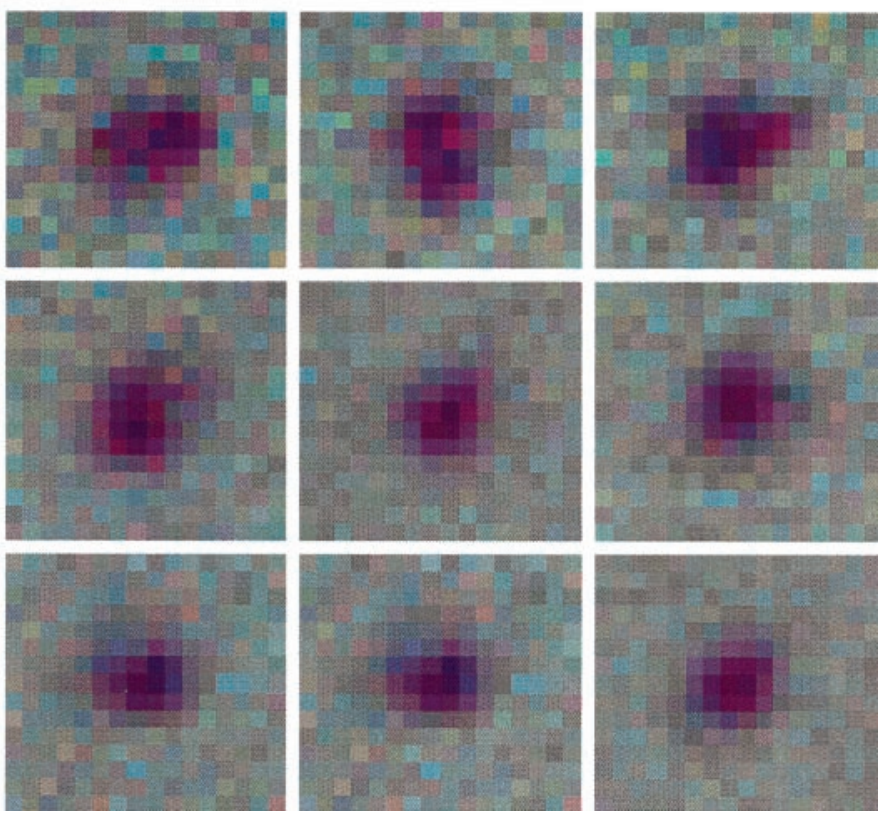

Figure 6. Receptive field size asymmetry. For nine L-ON (left) and nine L-OFF (right) cells recorded simultaneously, the average stimulus on the display 45 msec (3 frames, near time-to-peak) before a spike is shown in the same format as Figure 1. Cells of each group are sorted by RF size, from largest (top left) to smallest (bottom right). The RF location of each cell is different; these images have been cropped to the region immediately surrounding the RF.

A

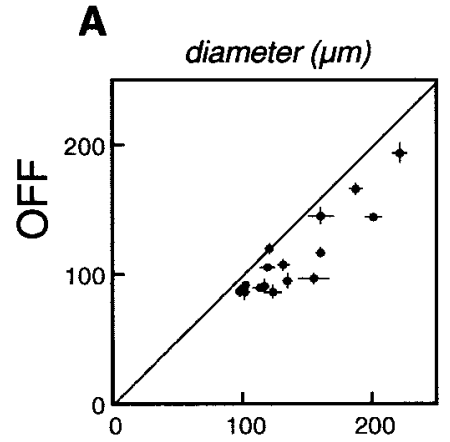

B

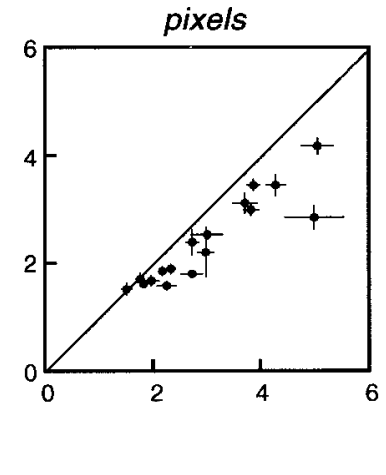

Figure 7. Receptive field size asymmetry summary. $A$, Each point shows the mean receptive field diameter of all L-ON cells and all L-OFF cells recorded in one preparation. Error bars, sometimes smaller than points, indicate 1 SEM. The diagonal line indicates equality for L-ON and L-OFF cells. Data from $169 \mathrm{~L}-\mathrm{ON}$ and $162 \mathrm{~L}-\mathrm{OFF}$ cells from 17 preparations are represented. $B$, Each point shows the square root of the mean number of pixels for L-ON and L-OFF cells for which the rms energy in the STA exceeded $25 \%$ of the rms energy of the strongest pixel.

rounds, generally weak in the present data (e.g., see Figs. 2, 6), were unlikely to complicate RF size estimates. The RF diameters shown in Figure $7 A$ were estimated from fits that included a surround the size and relative strength of which were free parameters; the size of the center is shown. Fits obtained without allowing for surrounds yielded similar results. Second, RF sizes measured without parametric models show the same asymmetry. For each cell, the number of pixels (spatial locations) at which the rms contrast in the STA exceeded $25 \%$ of the rms contrast of the strongest pixel was determined. The square root of this number (roughly proportional to diameter) is shown in Figure $7 B$ for

L-ON and L-OFF cells from the same preparations as Figure $7 A$. In all preparations, L-ON cell RFs on average contained a larger number of such pixels than L-OFF cell RFs $(p<0.001)$.

\section{Faster response kinetics in ON cells}

L-ON cells displayed consistently faster light responses than L-OFF cells. Figure 8 shows the STA time course (which can be interpreted as the time-reversed impulse response) of six L-ON and six L-OFF cells in one preparation. The primary lobe of the STA time course for the L-ON cells was visibly narrower than that of the L-OFF cells. This was quantified by examining the time of zero crossing, relative to the time of the spike, obtained from smooth parametric fits to time courses (see Materials and Methods). The mean time to zero crossing for $13 \mathrm{~L}-\mathrm{ON}$ cells recorded in this preparation was shorter $(62 \pm 1 \mathrm{msec})$ than for 10 L-OFF cells $(71 \pm 1 \mathrm{msec})$. A second example is shown in Figure 2. This kinetic asymmetry was consistent across preparations. Each point in Figure $9 B$ shows the mean and SEM of L-ON and L-OFF time to zero crossing in one preparation; data from 17 preparations are shown. In 16 of 17 preparations the points fall above the identity diagonal, indicating faster L-ON cell kinetics $(p<0.001)$. A linear regression indicates that L-OFF cells had, on average, $23 \%$ longer time to zero crossing than L-ON cells.

The time-to-peak of the STA was also usually shorter for L-ON cells. This was more difficult to observe in individual plots such as those in Figure 8, because of the coarse (15 or $8.33 \mathrm{msec}$ ) refresh interval of the stimulus display and consequent temporal sampling of the STA. However, averaged data from many cells and preparations reveal the trend clearly. Each point in Figure $9 A$ shows the mean and SEM of L-ON and L-OFF time-to-peak in one preparation. The points fall mostly above the identity diagonal, indicating faster L-ON cell kinetics $(p=0.006)$. A linear regression indicates that L-OFF cells had, on average, $13 \%$ longer time to peak. Finally, the time to trough (extreme point of 

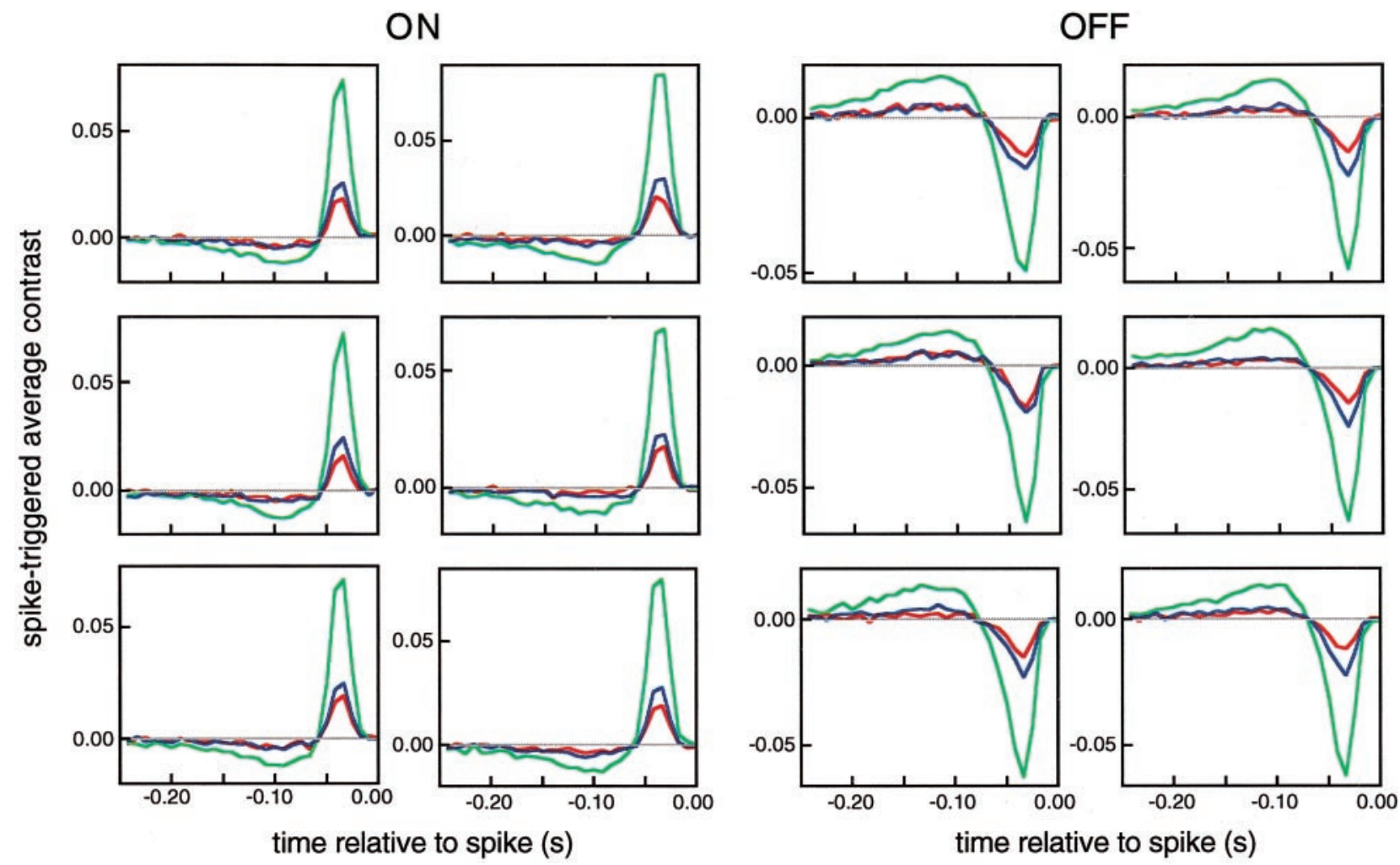

Figure 8. Kinetic asymmetry. STA time courses are shown for six L-ON (left) and six L-OFF (right) cells recorded simultaneously. Each panel shows the average time course of red, green, and blue display phosophor contrast in the $250 \mathrm{msec}$ preceding a spike, summed over the center of the RF, as in Figure 1.
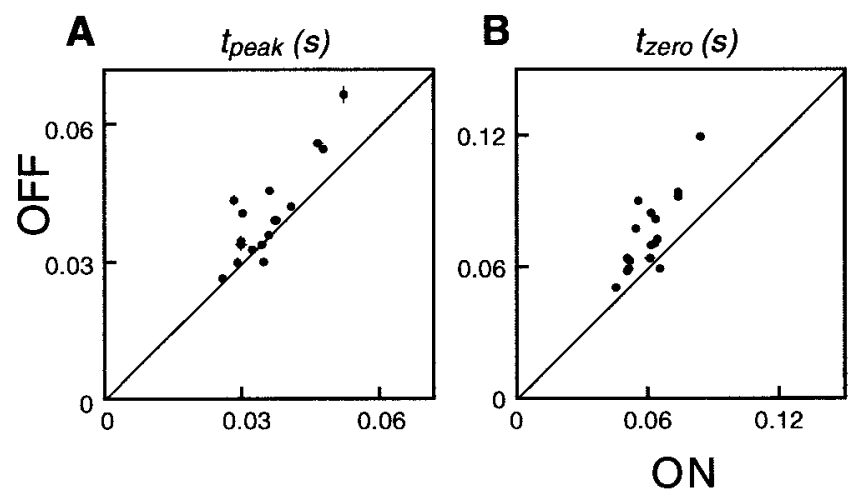

undershoot in STA time course) was on average $26 \%$ longer for L-OFF cells $(p<0.001)$, as can be seen in Figure $9 C$. Essentially identical results were obtained by measuring time-to-peak, zero crossing, and trough from the raw STA rather than parametric fits.

\section{More linear light responses in $\mathrm{ON}$ cells}

Although both L-ON and L-OFF cells displayed light response nonlinearity, the nonlinearity in L-OFF cells was more extreme. This is shown in the plots of Figure 10. Each panel shows spike rate as a function of the generator signal (stimulus weighted by STA, i.e., effective contrast) for one cell. For ON cells, incremental pulses of light correspond to positive generator signal, whereas decrements correspond to negative generator signal; for OFF cells the reverse holds. If light responses in RGCs were linear, these data would fall on straight lines. Both L-ON and L-OFF cells clearly displayed significant nonlinearities. However, over

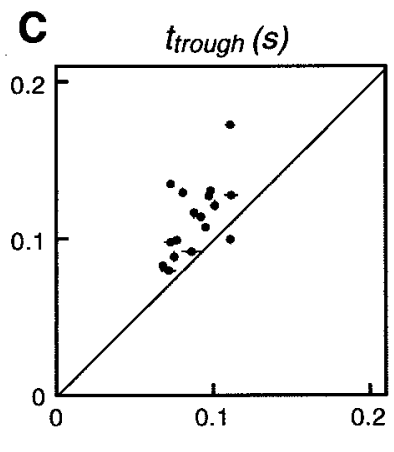

Figure 9. Kinetic asymmetry summary. $A$, Each point shows the mean time-to-peak of the STA time course for all L-ON cells and all L-OFF cells recorded in one preparation. Error bars indicate 1 SEM. Data from $169 \mathrm{~L}-\mathrm{ON}$ and $162 \mathrm{~L}-\mathrm{OFF}$ cells from 17 preparations are represented. $B$, Mean time to zero crossing for $\mathrm{L}-\mathrm{ON}$ and $\mathrm{L}-\mathrm{OFF}$ cells. $C$, Mean time to trough for $\mathrm{L}-\mathrm{ON}$ and L-OFF cells.

the stimulus range examined, L-OFF cells showed stronger rectification. This is evidenced by the sharp bend near zero for L-OFF cells compared with the more gentle curvature for L-ON cells. In fact, the L-OFF cell nonlinearity was nearly flat for negative generator values, whereas for L-ON cells it was not. This implies that L-ON cells provided graded responses to decrements of light, whereas L-OFF cells provided a limited representation of increments. A second example is shown in Figure 2.

This asymmetry was summarized by computing an index of nonlinearity, the logarithm of the ratio of the slope of the nonlinearity at maximum to the slope at zero. The mean nonlinearity index for $11 \mathrm{~L}-\mathrm{ON}$ cells was $0.1 \pm 0.02$ and for $8 \mathrm{~L}-\mathrm{OFF}$ cells was $1.1 \pm 0.05$ in the preparation of Figure 10 . This asymmetry was consistent across preparations. Each point in Figure $11 A$ shows the mean and SEM of the index of nonlinearity for all L-ON and L-OFF cells in each preparation. In 16 of 17 preparations, L-ON 
ON
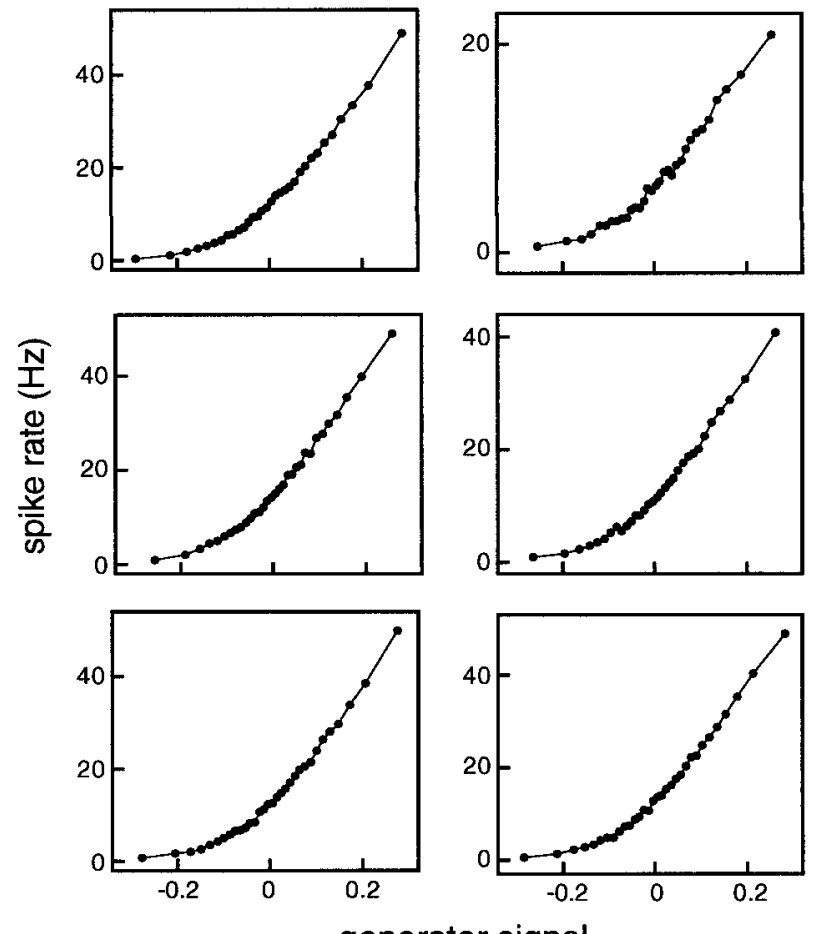
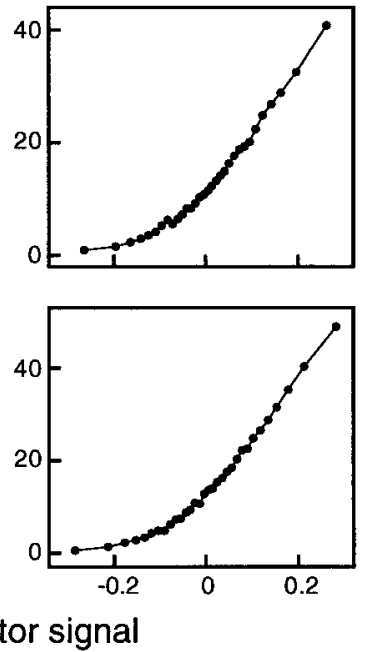

OFF
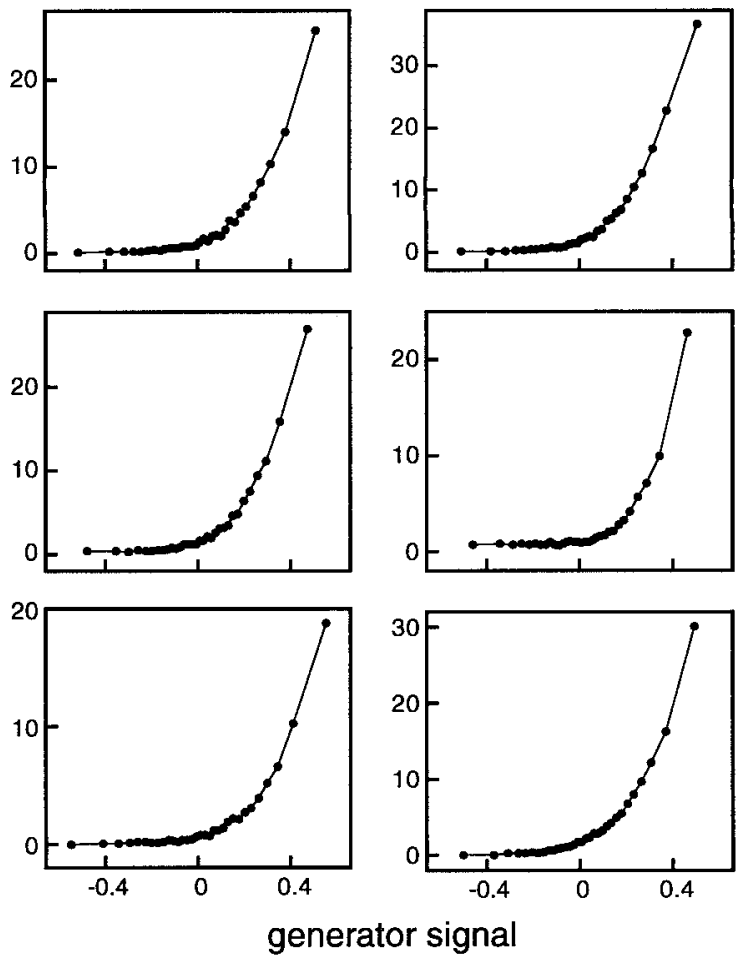

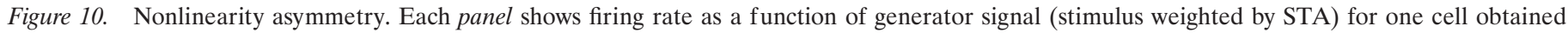
during white noise stimulation, as in Figure 1. Data are shown for six L-ON cells (left) and six L-OFF cells (right) recorded simultaneously.

Figure 11. Nonlinearity, gain, and SNR asymmetry summary. $A$, Each point shows the mean nonlinearity index for all L-ON cells and all L-OFF cells recorded in one preparation. Error bars indicate 1 SEM. Nonlinearity index is the logarithm of the ratio of the slope of the nonlinearity at the maximum generator signal value observed to the slope at zero generator signal. Data from $169 \mathrm{~L}-\mathrm{ON}$ and $162 \mathrm{~L}-\mathrm{OFF}$ cells from 17 preparations are represented. $B$, Mean logarithm of response gain for L-ON cells and L-OFF cells. Response gain is the derivative of firing rate (spikes per second) with respect to the contrast of a brief ( 15 or $8.33 \mathrm{msec}$ ) achromatic full-field flash de-

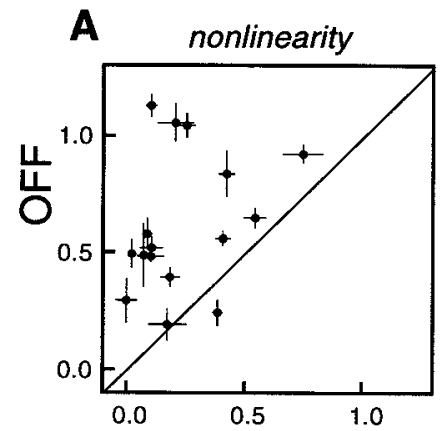

B

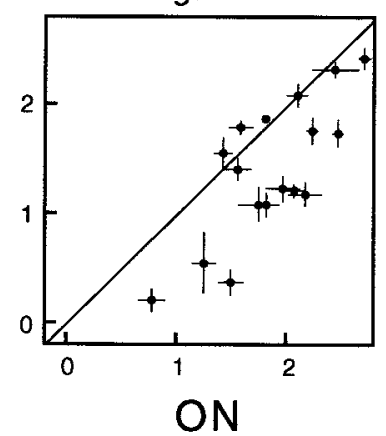

C

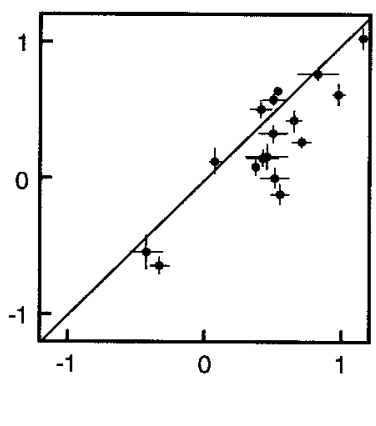

duced from white noise measurements. $C$, Mean logarithm of signal-to-noise ratio (SNR) for L-ON and L-OFF cells. SNR is defined as the response gain divided by the SD of spike counts observed at zero generator signal.

cells showed more linear light responses than L-OFF cells $(p<$ $0.001)$.

\section{Other properties}

The mean firing rate of L-ON and L-OFF cells during white noise stimulation varied between 5 and $30 \mathrm{~Hz}$ across preparations, but the firing rate of L-ON cells tended to exceed that of L-OFF cells (13 of 17 preparations; $p=0.005$ ). The firing rate asymmetry was also present at times when the white noise provided no net stimulation, i.e., when the generator signal was zero, equivalent to a steady uniform background (14 of 17 preparations; $p=0.002$ ), and thus was consistent with previous findings (Troy and Robson, 1992; Kremers et al., 1993) [but see Troy and Lee (1994), Benardete and Kaplan (1997, 1999)]. Color properties of L-ON and L-OFF cells were probed by comparing the mean ratio of the red to the green, or red to blue, phosphor contribution to the STA. No color asymmetry was observed $(p=0.13$ and $p=$ 0.79 , respectively), consistent with nonselective inputs from a random cone mosaic to both cell types.

\section{Consequences for visual sensitivity}

Asymmetries in RF size, kinetics, response nonlinearity, and firing rate could have significant consequences for the strength and fidelity of visual signals. Response gain (the derivative of spike rate with respect to the contrast of a full-field flash) was determined by multiplying the peak of the STA by the integration area of the RF and the slope of the nonlinearity at zero generator signal. Results are shown in Figure $11 \mathrm{~B}$. In most cases, L-ON cells displayed higher gain than L-OFF cells (14 of 17 preparations; $p=0.003)$. Asymmetry in response gain at the peak of the RF (gain divided by RF integration area) was less systematic (12 of 17 preparations; $p=0.013$ ). 
L-ON cells also provided a higher fidelity neural representation of weak full-field flashes. The signal-to-noise ratio (SNR) was estimated by dividing the response gain by the SD of spike counts observed at zero generator signal. The results in Figure $11 C$ show that L-ON cells usually had higher SNR than L-OFF cells ( 13 of 17 preparations; $p=0.006$ ), indicating a higher fidelity encoding of full-field stimuli. However, the peak SNR was not significantly greater for L-ON cells (8 of 17 preparations; $p=$ 0.79 ), indicating that the higher fidelity was attributable to larger RF size.

\section{DISCUSSION}

\section{Morphological types of cells recorded}

The significance of the ON-OFF asymmetries described here relies on the L-ON and L-OFF cells recorded in each preparation being of the same morphological class, e.g., parasol, because cells with different morphologies generally have different response properties. The L-ON and L-OFF cell groups had distinctive and similar response properties, tiled the retina, and were both well sampled by a common set of electrodes, suggesting that they are of the same morphological class. The RF size, response kinetics and gain, density, and sampling frequency of L-ON and L-OFF cells all suggest that they are parasol cells.

Two alternative possibilities could explain the consistent asymmetries observed here. (1) The recordings were heavily biased toward a single type of ON cell and a morphologically different type of OFF cell that have similar spatial and kinetic properties, whereas the opposite-sign cells of each type do not exist or were systematically excluded. (2) Different types of ON and OFF cells were sampled in different recordings, yet the RFs of the ON cell types recorded were systematically larger, the kinetics faster, and the nonlinearities milder than those of the OFF cell types recorded. These possibilities seem remote.

\section{Mechanisms of asymmetry}

The RF size asymmetry could be created by larger DFs in L-ON cells collecting inputs via bipolar cells from a larger region of the photoreceptor mosaic. Indeed, ON parasol and midget cells in human retina have DFs about 30 and 50\% larger than OFF parasol and midget cells, respectively (Dacey and Petersen, 1992). Also, ON $\alpha$ cells in rat (Peichl, 1989; Tauchi et al., 1992) and dog (Peichl et al., 1987) have larger DFs than OFF $\alpha$ cells. An alternative explanation might be that L-ON cells have stronger reciprocal excitatory connections that effectively mix the RFs of neighboring cells, as was reported for brisk transient cells in rabbit retina (DeVries, 1999). Such reciprocal excitation was not evident in the present recordings, and synchronized firing in neighboring cells (Chichilnisky and Baylor, 1999b), defined as the number of spikes synchronized within $\pm 5 \mathrm{msec}$ divided by the number expected by chance, was not systematically stronger in L-ON cells than L-OFF cells (8 of 17 preparations; $p=0.94$ ).

One potential source of kinetic asymmetry is the metabotropic glutamate receptor in ON-bipolars that inverts the sign of the light response from that in photoreceptors using a secondmessenger cascade (Nawy and Jahr, 1990). The extra biochemical steps involved in creating the ON-bipolar response, compared with the directly gated ionic currents underlying the OFF-bipolar response, might be expected to result in slower transfer of visual signals. Indeed, response latency, estimated as the time to $5 \%$ of the peak of the STA, was on average 10\% (1-2 msec) shorter for L-OFF cells than for L-ON cells (13 of 17 preparations; $p=$ $0.024)$. This asymmetry must be treated as tentative because it is small compared with the temporal discretization of the stimulus. Surprisingly, for the more reliably measured features of the light response - time to peak, zero crossing, and trough-L-ON cells displayed faster kinetics than L-OFF cells. This suggests that kinetic asymmetries in mechanisms responsible for later phases of the light response oppose and overwhelm those introduced at the photoreceptor synapse.

Larger ON RFs could shorten the time-to-peak if input from more photoreceptors caused a stronger light response that was followed by saturation. This would not explain the shorter time to zero crossing. Larger RFs could cause stronger adaptation to contrast (Shapley and Victor, 1981) or mean light level (EnrothCugell and Shapley, 1973), resulting in faster light responses [but see Cleland and Freeman (1988)]. This seems unlikely because in each preparation kinetics were highly stereotyped within L-ON and L-OFF populations (Fig. 8), although L-ON and L-OFF RF sizes typically varied by more than the mean difference between them (Figs. 3, 6). Alternatively, the mechanisms of adaptation in ON and OFF circuits may differ (Chander and Chichilnisky, 2001; Kim and Rieke, 2001). Another possibility is that the mechanisms that create the undershoot of the biphasic light response, perhaps in the inner retina, could counteract the primary lobe of the response sooner or more strongly in L-ON cells. Indeed, the mechanisms of inhibition differ for ON and OFF $\alpha$ cells in guinea pig (Demb et al., 2001).

There are at least two possible sources of asymmetries in nonlinearity. First, L-OFF cells could have a higher spike threshold relative to resting potential, raising the net stimulation required to enter a linear range of light response. Second, basal transmitter release rates could be lower in the bipolars that provide input to L-OFF cells, rectifying the response near zero contrast (Demb et al., 2001). Consistent with both possibilities, L-OFF cells usually displayed lower firing rates.

The higher fidelity (SNR) of L-ON responses was apparently caused by the integrated inputs from more photoreceptors and bipolars overwhelming sources of noise, because the SNR at the peak of the RF was not asymmetric. Surprisingly, the peak response gain in L-ON cells was at least as high as that in L-OFF cells. This is the reverse of the dependence of peak gain on receptive field size (eccentricity) reported in cat RGCs (Linsenmeier et al., 1982) that is attributable to denser dendritic branching in cells with smaller DFs (Kier et al., 1995). One possibility is that smaller L-OFF cell dendritic fields do not have correspondingly denser branching than nearby L-ON cells, but this interpretation is complicated by the significant effect of response nonlinearity on gain that was not accounted for in previous studies. Note that, as in previous studies, the present conclusions regarding gain and SNR depend strongly on the model for light response.

\section{Previous physiological findings}

One previous study described slower kinetics in OFF than ON color-opponent parvocellular-projecting RGCs (Lankheet et al., 1998), but others reported no obvious asymmetries between ON and OFF cells of the same functional class, including magnocellular-projecting (probably parasol) and parvocellularprojecting cells (Kremers et al., 1993; Benardete and Kaplan, 1997, 1999) and cat X and Y cells [but see Hammond (1974) and Linsenmeier et al. (1982)]. Several methodological differences could explain the lack of strong evidence for asymmetries in previous studies.

First, previous studies relied on sequential characterization of 
single cells. Variation in response properties over time or across animals could obscure ON-OFF asymmetries. Previous studies also examined cells over a range of eccentricities, rather than a collection of cells in a small area. Variability in RF size and response kinetics with eccentricity could obscure ON-OFF asymmetries; in the present data, this variation was often larger than the mean asymmetry observed within a preparation (Figs. 7, 9).

Second, previous studies used sinusoidally modulated grating stimuli and harmonic analysis to characterize spatial and temporal sensitivity assuming that RGC light responses are strictly linear, an approximation that may contribute to measurement error. The white noise method used here allows for instantaneous response nonlinearities such as spike generation or saturation, providing a more realistic description and empirically revealing significant nonlinearities (Fig. 2). Also, harmonic stimuli were not as completely interleaved as white noise, making the analysis less robust to adaptation and nonstationarities in recording, and circular symmetry of RFs was assumed in previous work; Figure 3 indicates that RFs often deviate from this assumption. These differences in analysis techniques might have introduced error in previous experiments that obscured ON-OFF asymmetries.

Finally, it is possible that differences between the in vitro preparation used here and the in vivo anesthetized preparations used in previous studies contributed to the discrepancy. Note that asymmetries were observed both in isolated retina and in RPEattached preparations.

\section{Asymmetries in central visual pathways}

Numerous psychophysical experiments have suggested asymmetries in visual sensitivity and perception that might reflect asymmetries in the ON and OFF pathways. For example, decrements are more easily detected than increments (Bowen et al., 1989; Kremers et al., 1993), and direction discrimination is more strongly dependent on spatial displacement for decrements than increments (Wehrhahn and Rapf, 1992). However, several factors complicate the interpretation of psychophysical findings in terms of ON and OFF neurons.

First, it is not clear how well ON and OFF cells can be selectively recruited by choice of visual stimuli. Typically, transient increments and decrements have been used to isolate the ON and OFF pathways, supported by evidence from pharmacological blockade of the ON pathway (Schiller et al., 1986), but L-ON cells clearly provide graded responses to decrements. Second, comparison of psychophysical and neurophysiological measurements requires quantitative detail. Although lower psychophysical detection thresholds for decrements could suggest higher sensitivity in OFF cells, the gain and SNR are higher for L-ON cells. The psychophysical asymmetry could instead result from decrements being encoded by both ON and OFF cells and increments being encoded primarily by ON cells. Also, if cells with smaller RFs are more closely spaced (Peichl, 1989), a larger number of OFF cells than ON cells may encode a given stimulus, offsetting higher SNR in individual ON cells. A third issue is that because many cell types at many stages of the visual pathways participate in the response to a stimulus, it is difficult to infer where psychophysical asymmetries arise. These asymmetries could reflect the existence of cell types for which no corresponding opposite-sign cells exist, rather than asymmetries between ON and OFF cells of the same morphological class.

These issues highlight the difficulty in interpreting attempts to compare the ON and OFF pathways in psychophysical experi- ments, but ultimately the significance of ON-OFF asymmetry depends on its consequences for visual behavior. The present results suggest that visual tasks which rely primarily on $\mathrm{ON}$ and OFF parasol cells should exhibit differences in spatial resolution, temporal resolution, and response as a function of contrast.

\section{REFERENCES}

Benardete EA, Kaplan E (1997) The receptive field of the primate P retinal ganglion cell, I: Linear dynamics. Vis Neurosci 14:169-185.

Benardete EA, Kaplan E (1999) The dynamics of primate M retinal ganglion cells. Vis Neurosci 16:355-368.

Bowen RW, Pokorny J, Smith VC (1989) Sawtooth contrast sensitivity: decrements have the edge. Vision Res 29:1501-1509.

Chander D, Chichilnisky EJ (2001) Adaptation to temporal contrast in primate and salamander retina. J Neurosci 21:9904-9916.

Chichilnisky EJ (2001) A simple white noise analysis of neuronal light responses. Network: Comput Neural Syst 12:199-213.

Chichilnisky EJ, Baylor DA (1999a) Receptive-field microstructure of blue-yellow ganglion cells in primate retina. Nat Neurosci 2:889-893.

Chichilnisky EJ, Baylor DA (1999b) Synchronized firing by ganglion cells in monkey retina. Soc Neurosci Abstr 25:1042.

Cleland BG, Freeman AW (1988) Visual adaptation is highly localized in the cat's retina. J Physiol (Lond) 404:591-611.

Croner LJ, Kaplan E (1995) Receptive fields of P and M ganglion cells across the primate retina. Vision Res 35:7-24.

Dacey DM (1994) Physiology, morphology and spatial densities of identified ganglion cell types in primate retina. Ciba Found Symp 184:1234, 63-70.

Dacey DM (2000) Parallel pathways for spectral coding in primate retina. Annu Rev Neurosci 23:743-775.

Dacey DM, Brace S (1992) A coupled network for parasol but not midget ganglion cells in the primate retina. Vis Neurosci 9:279-290.

Dacey DM, Petersen MR (1992) Dendritic field size and morphology of midget and parasol ganglion cells of the human retina. Proc Natl Acad Sci USA 89:9666-9670.

Demb JB, Zaghloul K, Haarsma L, Sterling P (2001) Bipolar cells contribute to nonlinear spatial summation in the brisk-transient (Y) ganglion cell in mammalian retina. J Neurosci 21:7447-7454.

DeVries SH (1999) Correlated firing in rabbit retinal ganglion cells. J Neurophysiol 81:908-920.

DeVries SH, Baylor DA (1997) Mosaic arrangement of ganglion cell receptive fields in rabbit retina. J Neurophysiol 78:2048-2060.

Drasdo N, Fowler CW (1974) Non-linear projection of the retinal image in a wide-angle schematic eye. Br J Ophthalmol 58:709-714.

Enroth-Cugell C, Shapley RM (1973) Flux, not retinal illumination, is what cat retinal ganglion cells really care about. J Physiol (Lond) 233:311-326.

Hammond P (1974) Cat retinal ganglion cells: size and shape of receptive field centres. J Physiol (Lond) 242:99-118.

Hartline HK (1938) The response of single optic nerve fibers of the vertebrate eye to illumination of the retina. Am J Physiol 121:400-415.

Kier CK, Buchsbaum G, Sterling P (1995) How retinal microcircuits scale for ganglion cells of different size. J Neurosci 15:7673-7683.

Kim KJ, Rieke F (2001) Temporal contrast adaptation in the input and output signals of salamander retinal ganglion cells. J Neurosci 21:287-299.

Korenberg MJ, Hunter IW (1986) The identification of nonlinear biological systems: LNL cascade models. Biol Cybern 55:125-134.

Kremers J, Lee BB, Pokorny J, Smith VC (1993) Responses of macaque ganglion cells and human observers to compound periodic waveforms. Vision Res 33:1997-2011.

Kuffler SW (1953) Discharge patterns and functional organization of mammalian retina. J Neurophysiol 16:37-68.

Lankheet MJ, Lennie P, Krauskopf J (1998) Distinctive characteristics of subclasses of red-green P-cells in LGN of macaque. Vis Neurosci 15:37-46.

Lee BB (1996) Receptive field structure in the primate retina. Vision Res 36:631-644.

Linsenmeier RA, Frishman LJ, Jakiela HG, Enroth-Cugell C (1982) Receptive field properties of $\mathrm{X}$ and $\mathrm{Y}$ cells in the cat retina derived from contrast sensitivity measurements. Vision Res 22:1173-1183.

Litke AM (1999) The retinal readout system: a status report. Nucl Instrum Methods Phys Res A 435:242-249.

Meister M, Pine J, Baylor DA (1994) Multi-neuronal signals from the retina: acquisition and analysis. J Neurosci Methods 51:95-106.

Nawy S, Jahr CE (1990) Suppression by glutamate of cGMP-activated conductance in retinal bipolar cells. Nature 346:269-271.

Packer OS, Williams DR, Bensinger DG (1996) Photopigment transmittance imaging of the primate photoreceptor mosaic. J Neurosci 16:2251-2260.

Peichl L (1989) Alpha and delta ganglion cells in the rat retina. J Comp Neurol 286:120-139. 
Peichl L, Ott H, Boycott BB (1987) Alpha ganglion cells in mammalian retinae. Proc R Soc Lond B Biol Sci 231:169-197.

Perry VH, Cowey A (1985) The ganglion cell and cone distributions in the monkey's retina: implications for central magnification factors. Vision Res 25:1795-810.

Perry VH, Silveira LC (1988) Functional lamination in the ganglion cell layer of the macaque's retina. Neuroscience 25:217-223.

Perry VH, Oehler R, Cowey A (1984) Retinal ganglion cells that project to the dorsal lateral geniculate nucleus in the macaque monkey. Neuroscience 12:1101-1123.

Polyak SL (1941) The retina. Chicago: University of Chicago.

Press WH, Flannery BP, Teukolsky SA, Vetterling WT (1988) Numerical recipes in C. Cambridge, UK: Cambridge University.

Rice JA (1988) Mathematical statistics and data analysis. Belmont, CA: Wadsworth.

Rodieck RW (1965) Quantitative analysis of cat retinal ganglion cell response to visual stimuli. Vision Res 5:583-601.

Sakai HM, Naka K, Korenberg MJ (1988) White-noise analysis in visual neuroscience. Vis Neurosci 1:287-296.

Schiller PH (1992) The ON and OFF channels of the visual system. Trends Neurosci 15:86-92.

Schiller PH, Sandell JH, Maunsell JH (1986) Functions of the ON and OFF channels of the visual system. Nature 322:824-825.
Shapley RM, Victor JD (1981) How the contrast gain control modifies the frequency responses of cat retinal ganglion cells. J Physiol (Lond) 318:161-179.

Silveira LC, Perry VH (1991) The topography of magnocellular projecting ganglion cells (M-ganglion cells) in the primate retina. Neuroscience 40:217-237.

Tauchi M, Morigiwa K, Fukuda Y (1992) Morphological comparisons between outer and inner ramifying alpha cells of the albino rat retina. Exp Brain Res 88:67-77.

Troy JB, Lee BB (1994) Steady discharges of macaque retinal ganglion cells. Vis Neurosci 11:111-118.

Troy JB, Robson JG (1992) Steady discharges of X and Y retinal ganglion cells of cat under photopic illuminance. Vis Neurosci 9:535-553.

Wassle H, Peichl L, Boycott BB (1981) Dendritic territories of cat retinal ganglion cells. Nature 292:344-345.

Watanabe M, Rodieck RW (1989) Parasol and midget ganglion cells of the primate retina. J Comp Neurol 289:434-454.

Wehrhahn C, Rapf D (1992) ON- and OFF-pathways form separate neural substrates for motion perception: psychophysical evidence. J Neurosci 12:2247-2250.

Werblin FS, Dowling JE (1969) Organization of the retina of the mudpuppy, Necturus maculosus. II. Intracellular recording. J Neurophysiol 32:339-355. 